\title{
Associations between folate intake, body composition, and liver status in healthy adults
}

\author{
Monika Młodzik-Czyżewska, Anna Malinowska and Agata Chmurzynska \\ Institute of Human Nutrition and Dietetics, Poznań University of Life Sciences, Poznań, Poland
}

\section{Abstract}

A link has recently been underlined between one-carbon metabolism and body weight and body composition, suggesting that folate may account for body mass determination and lipid metabolism. The aim of this study was thus to analyze whether folate intake is associated with body weight, body mass index (BMI), body fat percentage, and liver status.

409 subjects aged 20-40 were enrolled in Poznań, Poland from 2016 to 2018. Food intake was assessed using three-day food records. Folate intake was calculated based on food composition tables using the Diet 5.0 program (National Food and Nutrition Institute, Poland). Weight to $0.1 \mathrm{~kg}$ and height to $0.01 \mathrm{~m}$ were measured using an electronic scale and a stadiometer, respectively. BMI was calculated as body weight in kilograms divided by height in meters squared. Fat mass and lean body mass were determined using whole-body air-displacement plethysmography (BodPod, Cosmed, Italy). Genotyping of rs1801133 (MTHFR) was performed with TaqMan probes. The following biomarkers of liver steatosis were calculated: NAFLD liver fat score (NAFLD-LFS), fatty liver index (FLI), and hepatic steatosis index (HSI). To analyze the associations between folate intake and the measured parameters, we used multiple regression with adjustments for age, sex, and energy intake.

The mean body weight was $78.57 \pm 18.14 \mathrm{~kg}$, BMI $25.96 \pm 5.28 \mathrm{~kg} / \mathrm{m} 2$, and fat percentage $29.20 \% \pm 10.78 \%$. The median folate intake was $299.3 \mu \mathrm{g} /$ day. Dietary folate intake was negatively associated with body weight, BMI, and body fat percentage (p< 0.05 for all associations). Folate intake was also associated with fatty liver indices - namely HSI $(p<0.05)$ and FLI $(p<0.05)$. There was no association between folate intake and NAFLD-LFS. MTHFR rs1801133 polymorphism was not associated with any of the measured parameters.

Our findings suggest that folate intake may affect body weight and composition, as well as liver status. Higher folate intake could have a protective effect against obesity, but further studies are necessary to investigate the mechanism.

The authors declare that they have no conflict of interests.

This work was supported by the Polish National Science Centre

(grants 2014/15/B/NZ9/02134 and 2016/21/N/NZ9/01195).

\section{Conflict of Interest}

There is no conflict of interest. 